\title{
Protocol Deviation
}

National Cancer Institute

\section{Source}

National Cancer Institute. Protocol Deviation. NCI Thesaurus. Code C50996.

A variation from processes or procedures defined in a protocol. Deviations usually do not preclude the overall evaluability of subject data for either efficacy or safety, and are often acknowledged and accepted in advance by the sponsor. (CDISC Glossary) 